\title{
Percepciones de los residentes sobre el turismo deportivo: impactos, calidad de vida y apoyo al sector
}

\section{Residents' perceptions of sports tourism: impacts, quality of life and support for the industry}

\section{Percepções dos residentes sobre o turismo desportivo: impactos, qualidade de vida e apoio ao sector}

\author{
González-García, R.J. 1, Escamilla-Fajardo, P.2, López-Carril, S. 2, Nuñez-Pomar, J. 2 \\ ${ }_{1}$ Universidad Católica de Valencia San Vicente Mártir;2Universidad de Valencia
}

\begin{abstract}
RESUMEN
El sector del turismo deportivo recibe cada vez una mayor atención por parte de los distintos agentes sociales, teniendo un alto impacto en distintas esferas de la sociedad. El turismo no sólo afecta a las actitudes de los residentes hacia su desarrollo, sino también a su calidad de vida en general. Una vez que una comunidad o población se convierte en un destino turístico, la calidad de vida de los residentes locales se ve afectada por el desarrollo del mismo. Por consiguiente, es relevante conocer cuáles son las percepciones de los residentes respecto al impacto que causa el turismo deportivo. Por ello, el objetivo del presente estudio es analizar las percepciones de los residentes de Gran Canaria hacia los efectos del turismo deportivo, en ámbitos como su impacto, la calidad de vida y el apoyo al sector. Los resultados del estudio indican que, a mayores niveles de percepción positivos de los residentes sobre los impactos sociales, culturales, ambientales y económicos, hay un mayor apoyo hacia el desarrollo del turismo. Por otra parte, también se produce un efecto mediador significativo de la variable calidad de vida de los residentes, entre la relación de impactos percibidos y el apoyo al desarrollo del turismo activo y deportivo en Gran Canaria. Estos resultados posibilitan un mejor entendimiento de la perspectiva que los residentes tienen hacía el sector turístico deportivo, algo que puede ayudar a orientar futuras decisiones sobre la práctica, desarrollo y planificación del turismo deportivo.

Palabras clave: Percepciones residentes, turismo deportivo, calidad de vida, impacto social y apoyo.
\end{abstract}

\begin{abstract}
The sports tourism sector is receiving increasing attention from different social actors, having a high impact on different spheres of society. Tourism not only affects residents' attitudes towards their development, but also their quality of life in general. Once a community or population becomes a tourist destination, the quality of life of local residents is affected by its development. It is therefore important to know what residents' perceptions are of the impact of sports tourism. Therefore, the aim of this study is to analyse the perceptions of the residents of Gran Canaria towards the effects of sports tourism, in areas such as its impact, quality of life and support for the sector. The results of the study indicate that, at higher levels of positive perception by residents of the social, cultural, environmental and economic impacts, there is greater support for the development of tourism. On the other hand, there is also a significant mediating effect of the variable quality of life of
\end{abstract}




\section{González-García, R.J., Escamilla-Fajardo, P., López-Carril, S., Nuñez-Pomar, J.}

the residents, between the relationship of perceived impacts and the support for the development of active and sports tourism in Gran Canaria. These results make it possible to better understand the perspective that residents have towards the sports tourism sector, something that can help guide future decisions on the practice, development and planning of sports tourism.

Keywords: Sport Tourism, Resident perceptions, quality of life and social impact and support.

\section{RESUMO}

O sector do turismo desportivo está a receber cada vez mais atenção por parte de diferentes actores sociais, tendo um elevado impacto em diferentes esferas da sociedade. O turismo não afecta apenas as atitudes dos residentes em relação ao seu desenvolvimento, mas também a sua qualidade de vida em geral. Uma vez que uma comunidade ou população se torna um destino turístico, a qualidade de vida dos residentes locais é afectada pelo seu desenvolvimento. Por conseguinte, é importante conhecer a percepção que os residentes têm do impacto do turismo desportivo. Portanto, o objectivo deste estudo é analisar as percepções dos residentes da Gran Canária sobre os efeitos do turismo desportivo, em áreas como o seu impacto, qualidade de vida e apoio ao sector. Os resultados do estudo indicam que, em níveis mais elevados de percepção positiva dos residentes sobre os impactos sociais, culturais, ambientais e econômicos, há maior apoio para o desenvolvimento do turismo. Por outro lado, existe também um efeito mediador significativo da variável qualidade de vida dos residentes, entre a relação de impactos percebidos e o apoio ao desenvolvimento do turismo ativo e desportivo na Gran Canária. Estes resultados permitem compreender melhor a perspectiva que os residentes têm em relação ao sector do turismo desportivo, algo que pode ajudar a orientar futuras decisões sobre a prática, desenvolvimento e planeamento do turismo desportivo.

Palavras chave: Percepção de residente, turismo desportivo, qualidade de vida, o impacto social e apoio.

\section{INTRODUCCIÓN}

El crecimiento del turismo a nivel mundial, y la creencia que lo coloca como una de las industrias más importantes durante próximas generaciones, impulsa que se preste atención a los impactos derivados de la actividad y las consecuencias que este tiene en las comunidades receptoras. Debido a ese crecimiento, los gobiernos reconocen el turismo como una industria de futuro, esperando que los beneficios resultantes de esta actividad, sobrepasen los costes (Kaplanidou, 2012; Ma y Kaplanidou, 2017). La realidad, es que, a medida que el turismo afecta de manera local, regional o estatal, el grado de influencia de los impactos generado por este va aumentando, lo que permite que el turismo continúe creciendo internacionalmente (Nunkoo y Smith, 2013; Sharpley, 2014). Por lo tanto, se estima necesario dar un significado al desarrollo del turismo como medida para anticipar cambios e impactos, y redirigir consecuencias y conflictos que puedan causar el desarrollo de la actividad turística para la localidad. La actividad turística, concretamente la relacionada con la actividad física y el deporte, posee un importante potencial, que permite enriquecer las vidas de los residentes de la comunidad, proporcionando entre otras, oportunidades sociales y recreativas adicionales (Gibson 2017; Weed, 2008).

En ocasiones, estos impactos asociados al turismo plantean cuestiones sobre la capacidad de una localidad de acoger turistas, así como el crecimiento y evolución asociados a este hecho. Asimismo, según Wood (2011), estas cuestiones están relacionadas con la actividad turística desarrollada, las diferencias, tanto económicas como culturales entre los turistas y los residentes, o la capacidad de la comunidad de adaptarse a las peculiaridades turísticas sin quebrantar las actividades culturales. Estos impactos, además están influenciados por otros factores propios de cada comunidad. Entre ellas, destacan la influencia de algunas características sociodemográficas en las percepciones de los residentes como son la edad (Huh y Vogt, 2008; Sinclair-Maragh, 2017), el sexo (González-García, Parra, Añó y Calabuig, 2016; Nunkoo y Gursoy, 2012), la distancia de la residencia a la zona turística (Rasoolimanesh, Jaafar, Kock y Ramayah, 2015), el nivel de contacto de estos con los turistas (Andereck, Valentine, Knopf y Vogt, 2005; 


\section{Percepciones de los residentes sobre el turismo deportivo}

Wang y Pfister, 2008), el nivel académico de los residentes (Almeida-García, Balbuena-Vázquez y Cortes-Macías, 2015), el nivel de desarrollo turístico de la localidad (Plog, 2001), así como la dependencia económica de esta sobre la industria turística (Andereck y Nyaupane, 2011). Asimismo, también se han encontrado influencia de otras características sociodemográficas más específicas en las percepciones de los residentes sobre el turismo, como son el haber nacido en la comunidad (Sinclair-Maragh, 2017), el tiempo de residencia en la localidad (González-García et al., 2016; Gursoy, Chi y Dyer, 2010), o el grado de concentración turística en la zona (Hao, Long y Kleckley, 2011). Además, otros factores que condicionan los impactos asociados al turismo en una localidad son: la capacidad que tengan las comunidades de acogida de utilizar los recursos turísticos, el sentimiento de pertenencia hacia la comunidad, o el compromiso con esta (Fredline, 2005).

Por su parte (Bartolomé, Ramos y Rey-Maquieira, 2009) señalan que la repercusión que ejerza el turismo sobre las comunidades depende del tipo de turismo que se practique (individual, en grupo, itinerante, etc.), del motivo del viaje (cultural, de sol y playa, rural, o de aventura), así como el nivel de desarrollo económico de la localidad que acoge la actividad turística. Sin embargo, cabe resaltar, que no todo el mundo en una comunidad anfitriona percibe los impactos de turismo en la misma manera. A finales de la década de 1980, Krippendorf (1987) sugirió que las opiniones, creencias, y expectativas resultantes del turismo puede ser bastante diferentes dependiendo de la población general o grupo particular analizado. Por su parte, Chen y Raab (2009) descubrieron que los residentes generalmente no están tan informados sobre los diferentes tipos de turistas que visitan su localidad y por tanto no son capaces de ofrecer una opinión sobre los tipos de impactos que este pudiera ocasionar.

\section{Tipos de impacto}

Tal y como indica Chandralal (2010), los impactos asociados al turismo se han investigado, normalmente, desde una perspectiva económica y medioambiental, asimismo, han sido también frecuentemente investigados los impactos socioculturales asociados a esta actividad. Con todo ello, Williams y Lawson (2001) definen tres categorías de impacto: 1económico, 2-social y ambiental y 3-físico/mental. Por otro lado, autores coinciden en identificar los impactos económicos, físicos o del entorno y los impactos sociales (Ap y Crompton, 1998), así como los impactos medioambientales y culturales (AlmeidaGarcía et al., 2015; Látková y Vogt, 2012). Por su parte, Cooper (1995), señala que los impactos asociados al turismo se distinguen en: económicos, políticos, socioculturales, medioambientales y ecológicos. Otras investigaciones como la realizada por Kreag (2001) identifica siete tipologías de impacto asociados al turismo, que son las siguientes: económicos, medioambientales, socioculturales, de congestión, de servicios, de impuestos y actitudes de la comunidad. De manera similar, Ritchie, Burns y Palmer (2005) diferenció en siete tipos de impactos los asociados al turismo. Estos se clasifican en económico, físicos, socioculturales, psicológicos, políticos y turístico/comerciales. Por su parte, Fredline, Raybould, Jago y Deery (2005) clasifican estos impactos en económicos, medioambientales y sociales. Por otra parte, Hritz y Ross (2010) en su estudio sobre los impactos recibidos por los residentes en Indianápolis (EEUU) revelaron tras realizar el análisis factorial exploratorio que los impactos asociados al turismo, concretamente al turismo deportivo, pueden ser estructurados en cuatro factores: beneficios sociales, beneficios ambientales, beneficios económicos e impactos negativos generales.

\section{Percepciones sobre la calidad de vida}

La investigación relacionada con la calidad de vida es un campo de estudio emergente en las ciencias sociales, en las ciencias del comportamiento y en las ciencias ambientales y políticas durante las últimas décadas. Se considera una derivación del movimiento de indicadores sociales originado por primera vez en economía y sociología (Uysal, Sirgy, Woo y Kim, 2016). El ímpetu detrás del movimiento de los indicadores sociales se basa en la premisa de que las medidas económicas tradicionales del desarrollo social no pueden ser equiparadas con los indicadores más importantes del desarrollo que capturan el bienestar subjetivo (es decir, satisfacción con la necesidad, satisfacción de la vida, valor percibido o felicidad) según Woo, Uysal y Sirgy (2018). Basado en el análisis anterior, se puede argumentar que la calidad de vida se ha convertido en un tema de discusión importante en los últimos años (Uysal et al., 2016; Woo et al, 2018). 


\section{González-García, R.J., Escamilla-Fajardo, P., López-Carril, S., Nuñez-Pomar, J.}

Definir calidad de vida resulta complicado, puesto que se trata de una experiencia subjetiva que depende de las percepciones y sentimientos individuales (Uysal, Perdue y Sirgy, 2012). Señala Kim (2002), que calidad de vida se refiere a una satisfacción viviente y a los sentimientos de satisfacción o satisfacción con la propia experiencia en el mundo. Es lo que ven las personas, o lo que sienten sus vidas, sobre como los impactos afectan a la satisfacción de la vida individual o familiar, incluyendo la satisfacción con la comunidad, el vecindario y las circunstancias personales (Suess, 2014). Uysal et al., (2012), en el libro titulado "Handbook of Tourism and Quality-ofLife Research: Enhancing the Lives of Tourists and Residents of Host Communities", proponen que, dentro del pleno funcionamiento del sistema turístico, podemos encontrar dos modelos que deberían considerarse al examinar las actividades turísticas y sus consecuencias desde el punto de vista de los sistemas.

El primer modelo se centra en el impacto de las variables turísticas sobre el bienestar de los turistas. Específicamente, el enfoque clave en este modelo es cómo las experiencias de los turistas contribuyen a la calidad de vida, cómo se puede predecir la calidad de vida del turista a través de las características turísticas, las características del viaje, la satisfacción con la vida en general y el ciclo de vida del consumo (Uysal, et al., 2012). Seguidamente, se explica un segundo modelo que se centra en el impacto de las variables relacionadas con el turismo sobre el bienestar de los residentes de la comunidad anfitriona y otras partes interesadas. Este modelo describe cómo los residentes ven sus condiciones de vida, y cómo estas condiciones impactan el sentido de bienestar en varios condicionantes de vida, y la vida en general (Uysal et al., 2016; Woo, Kim y Uysal, 2015).

El turismo, no sólo afecta a las actitudes de los residentes hacia el desarrollo de este, sino también a su calidad de vida en general (Liburd, Benckendorff y Carlsen, 2012). La razón es que, una vez que una comunidad se convierte en un destino turístico, la calidad de vida de los residentes locales también se ve afectado por el desarrollo del mismo. Como tal, la calidad de vida de los residentes en una comunidad es una preocupación importante para los líderes comunitarios (Moscardo, 2012; Uysal, et al., 2016), y el propósito general de desarrollar el turismo se convierte en una meta para mejorar la calidad de vida de los residentes al abordar los beneficios económicos, sociales, culturales, recreativos, así como otros beneficios del turismo (Añó, 2011; Kerstetter y Bricker, 2012; Peters y Schuckert, 2014). Dentro del análisis sobre la calidad de vida de los residentes en comunidades turísticas, Uysal, et al. (2016) destacan que la investigación se ha dirigido dentro de tres categorías diferenciadas: (1) identificar a los mediadores entre el impacto del turismo y la calidad de vida; (2) comparar diferentes tipos de residentes comunitarios; y (3) investigar a los residentes.

Sobre la base del análisis anterior, se puede deducir que, en primer lugar, el impacto del desarrollo turístico juega un papel importante en la calidad de vida de los residentes de la comunidad (Andereck et al., 2005; Xu, Barbieri, Anderson, Leung y Rozier-Rich, 2016). En general, la investigación indica que el impacto del turismo tiene dimensiones tanto positivas como negativas. Esas dimensiones positivas contribuyen positivamente a mejorar la calidad de vida de los residentes, mientras que las dimensiones negativas, reducen la calidad de vida. Asimismo, aquellos que se benefician directamente del turismo a través del empleo, tienen más probabilidades de apoyarlo y reportar mayores niveles de calidad de vida en comparación con otros residentes no afiliados al turismo. Además, algunas investigaciones indican que las percepciones de los residentes sobre el impacto del turismo y su calidad de vida, pueden diferir entre los residentes atendiendo a diversas variables sociodemográficas tales como el tipo de residencia o el nivel de ingresos, el nivel de contacto con los turistas o si la ocupación de estos está relacionada con el turismo. También, las percepciones y actitudes de los residentes hacia el turismo, así como su calidad de vida, tienden a diferir dependiendo del nivel de desarrollo turístico, con la mayoría de los beneficios acumulados en las primeras etapas del desarrollo.

Tales resultados sugieren, que los residentes pueden percibir el desarrollo turístico como beneficioso para su calidad de vida. En esta línea, Andereck y Vogt (2000) encontraron que la calidad de vida era un predictor significativo de apoyo al desarrollo del turismo para algunas comunidades. Una mejora en la calidad de vida puede verse a través del desarrollo de productos turísticos que también pueden ser disfrutados por los residentes, como festivales, 


\section{Percepciones de los residentes sobre el turismo deportivo}

restaurantes, atracciones naturales y culturales, así como oportunidades de recreación al aire libre. También se puede ver a través de un mayor nivel de vida personal, a través de la creación de empleo, y el aumento del impuesto sobre la renta que resulta en un retorno de los servicios a los residentes (Andereck y Nyaupane, 2011).

En ese sentido, los encargados de la planificación, desarrollo y gestión gubernamental, así como los promotores comunitarios, han tomado cada vez más en cuenta la calidad de vida de los residentes a la hora de desarrollar políticas de desarrollo turístico (Andereck y Nyaupane, 2011). Por consiguiente, es el objetivo de este estudio analizar las percepciones de los residentes de Gran Canaria sobre los impactos asociados al desarrollo del sector turístico deportivo y su apoyo al desarrollo del mismo, atendiendo a la mediación que ejerce las percepciones sobre la calidad de vida de los residentes con respecto a esta relación.

\section{MATERIAL Y MÉTODOS}

\section{Participantes}

La muestra está compuesta por un total de 607 personas residentes en Gran Canaria. Gran Canaria tiene una población total de 843.195 habitantes (Instituto Nacional de Estadística, 2017). Según los datos publicados por el Instituto Nacional de Estadística, en el año 2017 había un total de 727.124 habitantes mayores de 16 años. La edad media de los encuestados fue de 36 años $(M=35,90 ; \mathrm{DT}=13,82)$ comprendidas entre los 18 y 86 años. Por su parte, más de la mitad de los encuestados $(50,50 \%)$ eran mujeres frente al 49,50\% de los encuestados son hombres.

\section{Instrumentos}

La obtención de información se utilizó como referencia diferentes escalas que miden las percepciones de los residentes hacia los impactos económicos, sociales, culturales, medioambientales, políticos-administrativos asociados al turismo deportivo (González-García, Añó-Sanz, ParraCamacho y Calabuig-Moreno, 2018). Asimismo, se utilizó una escala para medir las percepciones de los residentes sobre la calidad de vida, (Ma y Kaplanidou, 2017). También, se utilizó una escala diseñada para medir la predisposición a apoyar el desarrollo del turismo activo-deportivo por parte de los residentes (Lee, 2013). Todas estas escalas habían sido previamente validadas. La fiabilidad de las escalas se determinó mediante el análisis de Alfa de Cronbach, siendo en todas las escalas superior al punto de corte establecido en .70 (Hair, Anderson, Tatham y Black, 2006).

\section{Procedimiento}

La recogida de la muestra se llevó a cabo mediante un muestreo estratificado proporcional por cuota de sexo, donde la última unidad muestral ha sido el municipio. En este estudio, con una muestra de población de 727.124 residentes, el tamaño final de 607 residentes determinó un nivel de confianza del $95 \%$, con un margen de error del 3,9\%.

\section{Análisis estadístico}

Para el análisis de los datos, se utilizaron diferentes análisis estadísticos. En primer lugar, para todo lo referente a los estadísticos descriptivos (media, desviación típica, asimetría y curtosis) se utilizó el paquete estadístico SPSS (Statistical Package for the Social Science, Versión 24). También, se empleó la macro PROCESS, insertada en SPSS (versión 24), para observar la mediación entre las variable percepciones sobre la calidad de vida de los residentes. La interfaz PROCESS para SPSS (Hayes, 2013) permite conocer el porcentaje de varianza explicada de una variable dependiente, en función de un conjunto de variables independientes, midiendo la influencia (directa o indirecta) que efectúe una variable mediadora (Hayes, 2013; Fernandez-Muñoz y GarcíaGonzález, 2017).

Para probar el efecto mediador de las variables, se ha seguido el enfoque clásico de pasos causales propuesto por Baron y Kenny (1986). Los autores indican que para establecer la mediación deben cumplirse cuatro condiciones: en primer lugar, debe establecerse un vínculo directo entre la variable independiente y la variable dependiente; en segundo lugar, la variable independiente debe estar relacionada con la variable mediadora; en tercer lugar, el mediador debe estar significativamente relacionado con la variable dependiente cuando tanto la variable independiente como la mediadora son predictores de la variable dependiente; y en cuarto lugar, la relación entre la variable independiente y la variable dependiente debe reducirse significativamente cuando se añade el mediador. La mediación completa se 


\section{González-García, R.J., Escamilla-Fajardo, P., López-Carril, S., Nuñez-Pomar, J.}

establece cuando los coeficientes de trayectoria para la variable independiente en la primera condición son significativos y los mismos coeficientes no son significativos en la última condición. Asumiendo que las cuatro condiciones se cumplen, se apoya la mediación parcial (Baron y Kenny, 1986). Por su parte, Zhao, Lynch y Chen, (2010), realizan una revisión sobre los criterios establecidos por Baron y Kenny (1986), en el que formulan una serie de correcciones y a su vez, proporcionar un procedimiento y toma de decisiones a la hora de probar la mediación, clasificar su tipo e interpretar los resultados obtenidos. Los autores argumentan que los resultados de mediación (directa o indirecta) variaban según la significación entre las relaciones de las variables independientes, dependientes y mediadora, a través de la presencia o ausencia de mediación, sin necesidad de que deban mostrar previamente una relación estadísticamente significativa entre variables. Asimismo, para evaluar la importancia de los efectos mediadores se ha aplicado la prueba estadística z de
Sobel (Sobel, 1982). Si el valor de z es superior a 1,96 $(\mathrm{p}<0,05)$, se admite el efecto mediador.

\section{RESULTADOS}

$\mathrm{X}$ Con respecto a los resultados de esta investigación, en la tabla 1 se pueden apreciar las valoraciones medias obtenidas referentes a las percepciones de los residentes con respecto a las diferentes escalas analizadas. Observando la puntuación media del impacto económico es de 4.03 (DT=.83) sobre 5. Esto significa que los encuestados perciben con una disposición positiva el impacto económico que el turismo activo-deportivo genera en Gran Canaria. Atendiendo a los resultados se aprecia que el ítem más valorado es el 1, con una media de 4.12 (DT= .96). Así pues, los encuestados valoran con mayor apreciación la contribución en materia económica que recibe la comunidad gracias a las actividades desarrollas a través del turismo activo-deportivo

Tabla 1. Valoraciones medias, asimetría, curtosis y alfa de Cronbach de las escalas de impactos asociados a las percepciones de los residentes.

Cuestionario completo $(\alpha=.79)$.

Media (DT) Asimetría Curtosis

Impactos Económicos: $\alpha=.79$

El turismo deportivo aporta una mayor inversión económica a la comunidad

$4.12(.96) \quad-.94 \quad .40$

El turismo deportivo ayuda a mejorar la situación económica para muchos

residentes en esta comunidad

$3.89(1.04) \quad-.66 \quad-.28$

El turismo deportivo crea una oportunidad de mercado y atrae inversión extranjera

en Gran Canaria

$4.09(.96) \quad-.91 \quad .28$

Impactos Culturales: $\alpha=.74$

El turismo deportivo fomenta una variedad de actividades culturales y eventos para los residentes locales.

El turismo deportivo ayuda a mantener viva la cultura y ayuda a mantener la identidad étnica de los residentes locales.

El turismo deportivo ha dado lugar a un mayor intercambio cultural entre turistas y residentes siendo una experiencia enriquecedora

$3.65(1.07) \quad-.45 \quad-.49$

Impactos Político-administrativos $\alpha=.81$

La industria del turismo deportivo debe planificarse para el futuro

Los planes de desarrollo turístico-deportivo deben mejorarse continuamente

$3.33(1.18) \quad-.20 \quad-.80$

Creo que la isla debe hacer esfuerzo para atraer a más turistas deportivos

$3.84(1.02) \quad-.62--.21$

Impactos Sociales $\alpha=.78$

$\begin{array}{ccc}4.19(.90) & -.96 & .61 \\ 4.35(.86) & -1.40 & 1.84 \\ 4.23(.99) & -.86 & 1.61\end{array}$




\section{Percepciones de los residentes sobre el turismo deportivo}

\begin{tabular}{|c|c|c|c|}
\hline $\begin{array}{l}\text { El turismo deportivo genera problemas sociales como la delincuencia, consumo } \\
\text { de drogas... }\end{array}$ & $1.79(1.15)$ & 1.40 & .87 \\
\hline El turismo deportivo crea conflictos entre los residentes y visitantes & $1.97(1.16)$ & 1.03 & .07 \\
\hline $\begin{array}{l}\text { Los residentes sufren las consecuencias del turismo deportivo por vivir en una } \\
\text { zona de destino turístico }\end{array}$ & $2.36(1.23)$ & .54 & -.67 \\
\hline \multicolumn{4}{|l|}{ Impactos Medioambientales $\alpha=.82$} \\
\hline $\begin{array}{l}\text { El turismo deportivo provoca contaminación en el medio ambiente y acelera su } \\
\text { deterioro. }\end{array}$ & $2.21(1.23)$ & .71 & -.54 \\
\hline El turismo deportivo genera contaminación acústica (ruidos), del aire y del agua. & $2.25(1.20)$ & .62 & -.61 \\
\hline $\begin{array}{l}\text { Se necesitan normas ambientales reglamentarias para reducir los impactos } \\
\text { negativos del desarrollo del turismo deportivo. }\end{array}$ & $3.19(1.38)$ & -.22 & -1.14 \\
\hline $\begin{array}{l}\text { El turismo deportivo consume una gran cantidad de recursos naturales (agua, } \\
\text { energía...) }\end{array}$ & $2.65(1.24)$ & .24 & -.91 \\
\hline
\end{tabular}

Fuente. Elaboración propia. Nota. $\alpha=$ Alfa de Cronbach; DT= desviación típica

Por su parte, con respecto a una orientación positiva de las valoraciones, los impactos asociados a aspectos culturales en la comunidad son los menos valorados por la muestra, siendo la media de $3.60(\mathrm{DT}=.89)$ sobre cinco. De los tres ítems que forman esta dimensión, el mejor valorado es el seis, relacionado con la experiencia que supone la acogida de turistas y residentes a través del turismo deportivo $(\mathrm{M}=3.84$; $\mathrm{DT}=1.03)$. A éste, le siguen el fomento en la variedad de actividades culturales y eventos para los residentes $(\mathrm{M}=3.65 ; \mathrm{DT}=1.07)$, y por último la ayuda a mantener la cultura e identidad étnica de los residentes, con una media de 3.33 (DT=1.18) sobre cinco puntos.

Asimismo, por lo que respecta a los impactos políticoadministrativos, la escala formada por tres ítems presenta una media de 4.26 (DT= .78) sobre cinco. El ítem mejor valorado en la presente dimensión, afirma que los planes de desarrollo turístico-deportivo deben mejorarse continuamente $(\mathrm{M}=4.35$; $\mathrm{DT}=.86)$, siendo este ítem el que mejor valoración obtiene de toda la escala de impactos positivos. Los otros dos ítems que componen la escala obtienen puntuaciones elevadas (superiores a cuatro puntos).

Por lo que respecta a la dimensión social del coste obtiene una media de 2,04 sobre cinco. Con una media de 2.36 (DT=1.23), el ítem más alto de esta dimensión es el "debido al turismo deportivo se generan problemas sociales como delincuencia, consumo de drogas, etc." Según la muestra, en menor medida (M= 1.97; DT=1.17) se cree que los residentes sufren las consecuencias de vivir en una zona de destino turístico y con una media de $1.79(\mathrm{DT}=1.15)$ los residentes observan en menor medida que el turismo deportivo cree conflictos entre residentes y visitantes.

Por su parte, el coste medioambiental es según el presente estudio la dimensión en sentido negativo que mayor puntuación obtiene, con una media de 2.37 $(\mathrm{DT}=1.07)$ sobre cinco. El ítem medioambiental que más afecta es el que asegura que el turismo deportivo consume gran cantidad de recursos naturales $(\mathrm{M}=2.65$; $\mathrm{DT}=1.20)$ que igualmente es el más elevado del conjunto de los impactos. Asimismo, el ítem menos valorado de la escala $(M=2.21 ; \mathrm{DT}=1.23)$ es el relacionado con la contaminación y el deterioro del medio ambiente a causa del turismo deportivo.

Por su parte, en la tabla 2, se muestran los resultados obtenidos referentes a la escala que mide el apoyo de los residentes hacia el turismo activo-deportivo. El Alfa de Cronbach general de esta escala es de .81, lo que supone un buen dato de fiabilidad. Después de analizar las respuestas de los encuestados se obtiene una media de 3.97 sobre cinco, con una desviación típica (DT) de .79 en el global de la escala. El ítem con una media más elevada se apoya la idea de desarrollar el turismo de naturaleza en Gran Canaria ( $\mathrm{M}=4.31$; $\mathrm{DT}=.94)$. Asimismo, también obtiene una puntuación alta $(\mathrm{M}=4.22 ; \mathrm{DT}=.94)$ el ítem relacionado con el apoyo al desarrollo del turismo a través del desarrollo de eventos/programas/servicios turísticos deportivos. Por su parte, el ítem que hace referencia al apoyo de los residentes a que el turismo deportivo tenga un 


\section{González-García, R.J., Escamilla-Fajardo, P., López-Carril, S., Nuñez-Pomar, J.}

papel económico fundamental en la isla obtiene una valoración media de $4.09(\mathrm{DT}=.98)$. En el otro extremo, el ítem peor valorado en la escala $(\mathrm{M}=3.19$; DT=1.39) es el referente al apoyo hacia el desarrollo de turismo de golf.

Tabla 2. Valoraciones medias, asimetría, curtosis y alfa de Cronbach de la escala de apoyo al desarrollo del turismo activo -deportivo en la comunidad

\begin{tabular}{|c|c|c|}
\hline $\begin{array}{l}\text { Media } \\
\text { (DT) }\end{array}$ & Asimetrí & Curtosis \\
\hline $\begin{array}{l}\text { Me gustaría ver a más } \\
\text { turistas } \\
\text { actividades deportivas en }(1.01) \\
\text { Gran Canaria }\end{array}$ & -.851 & .246 \\
\hline $\begin{array}{l}\text { Apoyo a que el turismo } \\
\text { deportivo tenga un papel } 4.09 \\
\text { económico fundamental en (.99) } \\
\text { Gran Canaria }\end{array}$ & -.995 & .551 \\
\hline $\begin{array}{l}\text { Apoyo al desarrollo de } \\
\text { eventos/programas/servicios } \\
\text { turísticos-deportivos (por } \\
\text { ejemplo: instalaciones de } 4.28 \\
\text { recreo, }(.93) \\
\text { acontecimientos deportivos, } \\
\text { eventos, etc.) }\end{array}$ & -1.32 & 1.345 \\
\hline $\begin{array}{l}\text { En general, yo apoyo el } \\
\text { desarrollo del turismo a } 4.00 \\
\text { través del turismo de }(.98) \\
\text { actividades acuáticas }\end{array}$ & -.852 & .273 \\
\hline $\begin{array}{l}\text { En general, yo apoyo el } 3.21 \\
\text { desarrollo del turismo de golf } 3.43) \\
\text { en la isla }\end{array}$ & -.231 & -1.22 \\
\hline $\begin{array}{l}\text { En general, yo apoyo el } \\
\text { desarrollo del turismo de } \\
\text { naturaleza en la isla } 4.35 \\
\text { (senderismo, } \\
\text { cicloturismo...) }\end{array}$ & -1.68 & 2.93 \\
\hline $\begin{array}{l}3.99 \\
(.77)\end{array}$ & -.99 & 1.41 \\
\hline
\end{tabular}

Fuente. Elaboración propia. Nota. $\alpha=$ Alfa de Cronbach; DT= desviación típica

Seguidamente, en la tabla 3, se muestran los resultados obtenidos referentes a la escala que mide las percepciones sobre la calidad de vida de los residentes hacia el turismo activo-deportivo. El Alfa de Cronbach general de esta escala es de .75, lo que supone un buen dato de fiabilidad. Después de analizar las respuestas de los encuestados se obtiene una media de 3.71 sobre cinco, con una desviación típica (DT) de .78 en el global de la escala. El ítem con una media más elevada se apoya en la satisfacción con la localidad de residencia $(\mathrm{M}=4.09 ; \mathrm{DT}=.99)$. Asimismo, también obtiene una puntuación alta $(\mathrm{M}=3.96 ; \mathrm{DT}=.94)$ el ítem relacionado con la satisfacción general con su calidad de vida. Por su parte, el ítem que hace referencia al futuro prometedor/esperanzador de la comunidad obtiene una valoración media de 3,44 (DT=1.01). En el otro extremo, el ítem peor valorado en la escala $(\mathrm{M}=3.35 ; \mathrm{DT}=1.14)$ es el referente a la mejora de la calidad de vida de los residentes mediante el turismo deportivo.

Tabla 3. Valoraciones medias, asimetría, curtosis y alfa de Cronbach de la escala relacionada con percepciones sobre la calidad de vida de los residentes.

\begin{tabular}{|c|c|c|c|}
\hline Calidad de Vida $(\alpha=.75)$ & $\begin{array}{c}\text { Media } \\
\text { (DT) }\end{array}$ & Asimetría & Curtosis \\
\hline $\begin{array}{l}\text { En general, estoy satisfecho } \\
\text { con mi calidad de vida }\end{array}$ & $\begin{array}{l}3.96 \\
(.94)\end{array}$ & -.73 & .20 \\
\hline $\begin{array}{l}\text { Estoy satisfecho con mi } \\
\text { localidad como lugar para } \\
\text { vivir }\end{array}$ & $\begin{array}{l}4.09 \\
(.99)\end{array}$ & -1.00 & .61 \\
\hline $\begin{array}{l}\text { El turismo deportivo ha } \\
\text { mejorado la calidad de vida } \\
\text { de los residentes }\end{array}$ & $\begin{array}{c}3.35 \\
(1.14)\end{array}$ & -.29 & -.61 \\
\hline $\begin{array}{l}\text { El futuro de la comunidad } \\
\text { es } \\
\text { prometedor/esperanzador }\end{array}$ & $\begin{array}{c}3.44 \\
(1.01)\end{array}$ & -.24 & -.32 \\
\hline Total & $\begin{array}{l}3.71 \\
(.78) \\
\end{array}$ & -.65 & -57 \\
\hline
\end{tabular}

Fuente. Elaboración propia. Nota. $\alpha=$ Alfa de Cronbach; DT= desviación típica

Análisis mediador

En cuanto a los resultados del análisis mediador, en primer lugar, se presentan los resultados del análisis de regresión lineal simple entre las variables independientes (impactos asociados a las percepciones sobre el turismo deportivo), la variable mediadora (percepción sobre la calidad de vida de los residentes), y la variable dependiente (apoyo al desarrollo del turismo deportivo).

Tal y como se observa en la figura 1, existe una relación significativa $(p \leq 0,05)$ entre todas las 


\section{Percepciones de los residentes sobre el turismo deportivo}

variables analizadas exceptuando la relación entre los impactos medioambientales percibidos por los residentes y sus percepciones sobre la calidad de vida.

Atendiendo a los resultados obtenidos por el efecto mediador de la variable calidad de vida, se puede observar como los coeficientes estandarizados de todas las dimensiones son estadísticamente significativos exceptuando la relación entre los impactos medioambientales y las percepciones sobre la calidad de vida de los residentes. El efecto indirecto que la variable mediadora calidad de vida en relación a la dependiente (apoyo) y las variables independientes impactos económicos $(\beta=.15$, $\mathrm{IC}=.11-.20), \quad$ impactos culturales $(\beta=.14$, $\mathrm{IC}=.10-.18)$, impactos políticos $(\beta=.12, \mathrm{IC}=.08-.16)$, impactos sociales $(\beta=-.04, \mathrm{IC}=-.08-.00)$ e impactos medioambientales $(\beta=-.03, \mathrm{IC}=-.06-.00)$.
Tabla 4. Efecto indirecto relacionado a la variable mediadora calidad de vida con respecto a los impactos y el apoyo al desarrollo del turismo activo-deportivo en la comunidad.

\begin{tabular}{lccc}
\hline \hline Efecto indirecto & Estimado & ICi & ICs \\
\hline EC. $\rightarrow$ CV $\rightarrow$ AP & $.15^{*}$ & .11 & .20 \\
CU. $\rightarrow$ CV $\rightarrow$ AP & $.13^{*}$ & .10 & .18 \\
PO. $\rightarrow$ CV $\rightarrow$ AP & $.12^{*}$ & .08 & .16 \\
SO $\rightarrow$ CV $\rightarrow$ AP & $-.04^{*}$ & -.07 & -.00 \\
ME. $\rightarrow$ CV $\rightarrow$ AP & -.03 & -.06 & .01 \\
\hline \hline
\end{tabular}

Nota: IE.= Económicos; IC.$=$ Culturales; IP.= Políticos-administrativos; IS.= Sociales; IM.= Medioambientales; $\mathrm{AP}=$ Apoyo; $\mathrm{CV}=$ Calidad de Vida; ICi= Intervalo de confianza inferior; ICs= Intervalo de confianza superior.

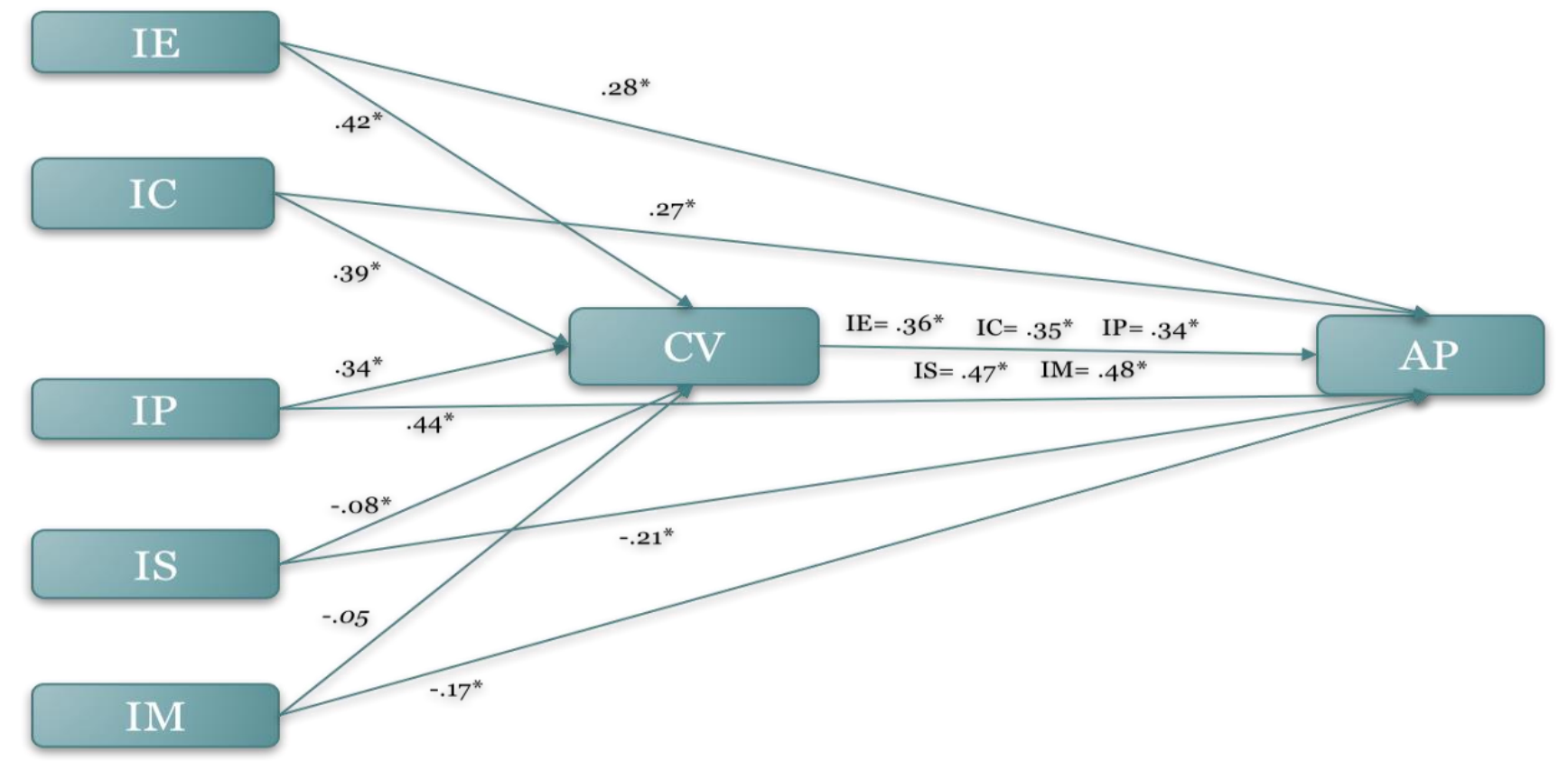

Figura 1. Coeficientes de regresión entre variables independientes, mediadoras y dependientes.

El efecto total de los impactos asociados a las percepciones de los residentes sobre el apoyo al desarrollo del turismo deportivo resulta significativo en toda las variables: impactos económicos $(\mathrm{R} 2=.21$, $\mathrm{F}=162.17, \mathrm{p}<0.001, \mathrm{Ef}=.43, \mathrm{IC}=.37-.50)$, impactos culturales $(\mathrm{R} 2=.21, \mathrm{~F}=164.74, \mathrm{p}<0.001, \mathrm{Ef}=.40$, $\mathrm{IC}=.34-.47)$ impactos políticos $(\mathrm{R} 2=.30, \mathrm{~F}=272.32$, $\mathrm{p}<0.001, \mathrm{Ef}=.54, \mathrm{IC}=.49-.62)$, impactos sociales $(\mathrm{R} 2=.09, \mathrm{~F}=65.09, \mathrm{p}<0.001, \mathrm{Ef}=-.25, \mathrm{IC}=-.31-$ -.19) e impactos medioambientales $(\mathrm{R} 2=.07, \mathrm{~F}=$ $46.63, \mathrm{p}<0.001, \mathrm{Ef}=-.20, \mathrm{IC}=-.25--.14)$.

Tabla 5. Efecto total relacionado a la variable mediadora calidad de vida con respecto a los impactos 


\section{González-García, R.J., Escamilla-Fajardo, P., López-Carril, S., Nuñez-Pomar, J.}

y el apoyo al desarrollo del turismo activo-deportivo en la comunidad.

\begin{tabular}{lccc}
\hline \hline Efecto Total & Estimado & ICi & ICs \\
\hline EC. $\rightarrow$ AP & $.43^{*}$ & .37 & .50 \\
CU. $\rightarrow$ AP & $.40^{*}$ & .34 & .47 \\
PO. $\rightarrow$ AP & $.56^{*}$ & .49 & .62 \\
SO. $\rightarrow$ AP & $-.25^{*}$ & -.31 & -.19 \\
ME. $\rightarrow$ AP & $-.20^{*}$ & -.25 & -.14 \\
\hline \hline
\end{tabular}

Nota: IE. $=$ Económicos; IC.$=$ Culturales; IP.= Políticos-administrativos; IS.= Sociales; IM.= Medioambientales; $\mathrm{AP}=$ Apoyo; $\mathrm{IC} \mathrm{i}=$ Intervalo de confianza inferior; ICs= Intervalo de confianza superior.

También se muestran los resultados del test de Sobel, que reflejan que el efecto de mediación parcial descrito en el modelo ha sido estadísticamente significativo (EC: $\mathrm{z}=7.57, \mathrm{p}<0,001 ; \mathrm{CU}: \mathrm{z}=7.46$, $\mathrm{p}$ $<0,001$; PO: $z=6.78, p<0,001$; SO: $z=-2.45, p<$ $0,001 ;$ ME: $\mathrm{z}=-1.98, \mathrm{p}>0,05)$.

\section{DISCUSIÓN}

La relación entre las variables asociadas a las percepciones de los residentes en las comunidades donde se desarrollan actividades turísticas y su relación directa sobre el apoyo al desarrollo de estas en comunidad han sido ampliamente estudiadas, afirmando la existencia de impactos tanto negativos como positivos (Brida, Del Chiappa, Meleddu y Pulina, 2014; González-García et al., 2016, Parra, Añó, Calabuig y Ayora, 2016). Por otra parte, se distingue la importancia de otro tipo de variables como son la calidad de vida y su relación con los impactos asociados al turismo, a menudo analizadas sin ser vinculadas en la relación de los impactos asociados a la actividad, con el apoyo al desarrollo de esta en la comunidad.

La bibliografía sugiere que existe una relación positiva entre los factores asociados a los impactos positivos percibidos por los residentes y el apoyo que estos presentan hacia el desarrollo turístico. Estos reflejan la visión común del turismo, como una herramienta para la prosperidad económica y el desarrollo de la comunidad. Los resultados obtenidos concuerdan con los hallazgos de Stylidis, Biran, Sit y Szivas (2014), que señalan que cuando los residentes obtienen una mejor percepción sobre los impactos sociales, culturales, ambientales y económicos positivos, estos apoyan el desarrollo del turismo. Por su parte, Yu, Chancellor y Cole (2011) señalan en su estudio que, en los Estados Unidos, las percepciones de los residentes sobre los impactos socioculturales y económicos positivos afectan positivamente el apoyo al desarrollo turístico. Por su parte, Wang y Pfister (2008) señalan que los impactos positivos derivados del desarrollo turístico serán aquellos con incidencia directa en la economía (incremento de las oportunidades de empleo; mejora de la inversión, más desarrollo y mejores infraestructuras; y mejora de los ingresos y nivel de vida). Asimismo, tal y como señala Müller (2012), existen diferentes grados de percepción entre los impactos. Por otro lado, los efectos que las percepciones de los residentes pudieran tener en el apoyo al desarrollo del turismo en la comunidad variarían, siendo estos efectos más fuertes en los impactos positivos, y más reducidos, en los impactos negativos (González-García et al., 2016; SinclarMaragh y Gursoy, 2016).

Como apunta Colmenares (2008), la calidad de vida de los residentes está estrechamente relacionada con las percepciones positivas y negativas de los impactos asociados al turismo. Asimismo, estos impactos afectan de manera directa a la calidad de vida de los residentes (Ko y Stewart, 2002). También, siguiendo la línea anterior, Twynam y Johnston (2004) señalan el aumento de la calidad de vida del residente como condicionante para la obtención de apoyo por parte de estos al desarrollo del turismo en la comunidad. Estudios previos han encontrado que las percepciones de los residentes sobre su satisfacción con la calidad de vida en la comunidad están directamente relacionadas con el apoyo a la actividad turística por parte de los residentes (Kaplanidou et al., 2013). Por su parte, Nunkoo y So (2016) no manifestaron que la relación entre la calidad de vida de los residentes, y el apoyo hacia el desarrollo del turismo, se llevara a cabo. Asimismo, se debe puntualizar que los impactos positivos asociados a la actividad turística influyeron significativamente con la calidad, de vida mientras que los impactos negativos no lo hicieron (Kaplanidou et al., 2013; Nunkoo y So, 2016).

En nuestro estudio, se puede observar como el efecto mediador de calidad de vida de los residentes en la relación entre los impactos asociados a la actividad turístico-activo-deportiva y el apoyo al desarrollo del 


\section{Percepciones de los residentes sobre el turismo deportivo}

turismo en la comunidad, es significativo en todas las variables, exceptuando en los impactos medioambientales.

Cabe destacar que estos impactos están en sentido negativo, lo que estaría en concordancia con los trabajos de Nunkoo y So (2016), quienes observaron que aquellas variables referentes a los impactos negativos de la actividad turística no intervenían significativamente en el modelo. Este hecho puede deberse a que, incluso si un individuo siente que la actividad turística influye considerablemente en su comunidad, si este no siente que esta actividad es personalmente importante, es poco probable que el individuo atribuya el efecto del impacto del turismo en la comunidad (Andereck y Nyaunpane, 2011). En este sentido, el residente en Gran Canaria percibe que el impacto del turismo activo-deportivo es importante para su calidad de vida, pero a su vez, no percibe que los impactos medioambientales asociados a la actividad vayan a influir en su calidad de vida. Por otro lado, los resultados con respecto al resto de escalas sí resultan significativos en nuestro estudio, incluyendo la variable impacto social, que junto a la de impactos medioambientales, se desarrollaban en sentido negativo.

Señalan Gursoy, Yolal, Ribeiro y Panosso Netto (2017), que, dado que los pasos para la licitación y la planificación tienen un mínimo componente de participación ciudadana, el nivel de confianza y percepción que los residentes puedan tener hacia los responsables en la organización y gestión del sector, juega un papel significativo. De esta manera, estudios como el elaborado por Woosnam y Aleshinloye (2018), señalan que las organizaciones encargadas de la gestión y desarrollo de la actividad turística, necesitan aprovechar esta relación entre residentes e instituciones, diseñando programas donde los locales puedan sentirse integrados en actividades de ocio junto con los turistas.

Las variantes en cuanto a los resultados de los diferentes estudios pueden deberse al tipo de actividad analizada en cada uno de ellos. Por un lado, Kaplanidou et al. (2013) centró su análisis en los mega eventos deportivos, cuyas características sí que interfieren directamente en la calidad de vida de los residentes, sobre todo durante el tiempo de realización del mismo. Por otro lado, Nunkoo y So (2016) en su análisis, señalan que la calidad de vida únicamente pone énfasis en las consecuencias positivas asociadas a la actividad turística, puesto que esta es vista como una industria que mejora significativamente la calidad de vida de las comunidades (Kim, Uysal y Sirgy, 2013).

Asimismo, estudios como el de Ma y Kaplanidou (2017) probaron el efecto mediador de la calidad de vida, y apuntaron que la calidad de vida si influye significativamente en el apoyo al evento. De esta manera, Andereck y Nyaunpane (2011) señala que el efecto mediador de la calidad de vida por parte de los residentes de una comunidad se percibía especialmente desde una perspectiva económica.

\section{CONCLUSIONES}

Por lo tanto, los resultados de los distintos estudios confirman que la calidad de vida ejerce un efecto mediador entre la percepción de los residentes sobre los impactos asociados a turismo, y su disposición a apoyar el desarrollo del mismo, siempre que perciban que los beneficios asociados superen a los costes. De todas formas, se debe tener en cuenta el contexto donde se realiza el estudio, puesto que, tal y como se ha argumentado, estos resultados podrían variar. Considerando la relación entre los impactos percibidos por los residentes, y el efecto mediador de variables como la calidad de vida de los residentes, se concluye que el efecto mediador de la variable calidad de vida de los residentes es significativo entre la relación de impactos percibidos, y el apoyo al desarrollo del turismo activo deportivo en Gran Canaria.

\section{APLICACIONES PRÁCTICAS}

El presente estudio ofrece una disposición para la práctica, el desarrollo y la planificación del turismo. En primer lugar, contribuye a la planificación local, ayudando a los encargados en esta labor a centrarse en el desarrollo de la actividad, con el objetivo de conservar, mejorar y que esta sea compatible con la imagen de la localidad. Los organismos encargados en la planificación y gestión deben considerar el posible efecto que estas políticas tienen en los residentes, ya que pueden surgir discrepancias con los intereses entre instituciones y ciudadanos. Por lo tanto, este tipo de estudio contribuye a una mejor compresión del punto de vista que tiene el residente hacia el sector. 


\section{González-García, R.J., Escamilla-Fajardo, P., López-Carril, S., Nuñez-Pomar, J.}

Los programas de participación ciudadana en la planificación turística son escasos. En este sentido, los gobiernos tienen la necesidad de crear formas de involucrar a los residentes en las tomas de decisiones puestos que esta afecta directamente al desarrollo de la sociedad. Estos, deben darse cuenta que la plena participación de los residentes no interfiere en los procesos de planificación, sino que los mejora. Involucrar a los residentes en la concreción de la visión, las metas y las políticas comunitarias pueden aumentar la calidad de vida de estos. Para ello este proceso requiere informar, educar y capacitar a los residentes, encargados de la planificación pública y empresas locales en aumentar la compresión de los ciudadanos. Si los organismos encargados en la planificación y gestión no son capaces de involucrar a los residentes, no se puede garantizar el éxito y la sostenibilidad del turismo.

Por otro lado, desde un punto de vista del marketing, involucrar a la comunidad local antes de emprender acciones turísticas, posibilita conocer las preferencias y actitudes que estos tienen sobre diferentes tipos de turismo. Este hecho permite dilucidar la importancia que tienen la diversificación turística en una comunidad, alejándose del concepto clásico de turismo de sol y playa. De la misma manera, permite considerar el aprovechamiento de las diferentes infraestructuras que actualmente existen en la comunidad con capacidad para la acogida de la actividad turística.

Como limitaciones del estudio, la primera tiene vínculo con el momento temporal de la obtención de los datos, desarrollándose la fase de campo durante el periodo de temporada alta de Gran Canaria. Este hecho puede suponer un elemento condicionante respecto a las percepciones que tienen los residentes sobre el turismo, por lo que debe ser tenido en cuenta a la hora de posibles extrapolaciones de los resultados. Otra limitación que identificamos en el estudio está relacionada con los ítems utilizados. Una vez analizados los datos, se considera que podría haber sido interesante haber adaptado otro tipo de cuestionarios, más allá de los utilizados, de cara a completar todavía más las dimensiones analizadas.

De cara a futuros estudios sería conveniente analizar otro tipo de variables que afecten a la integración entre los impactos y el apoyo por parte de los residentes. También sería importante analizar si existen otro tipo de variables que ejerzan como mediador en este tipo de interacción. Por último, sería interesante extrapolar el tipo de análisis realizado en este estudio a otras poblaciones para ver si se obtienen resultados similares.

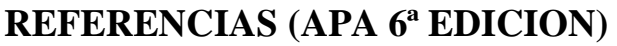

1. Bay-Hinitz, A.K., Peterson, R.F. y Quilitch, H.R. (1994). Cooperative games: A way to modify aggressive and cooperative behaviors in young children. Journal of Applied Behavior Analysis, 27(3), 435-446.

2. Almeida-García, F., Balbuena-Vázquez, A., y CortesMacías, R. (2015). Resident's attitudes towards the impacts of tourism. Tourism Management Perspectives, $13, \quad 33-40 . \quad$ DOI: https://doi.org/10.1016/j.tmp.2014.11.002

3. Andereck, K. L., y Nyaupane, G. P. (2011). Exploring the nature of tourism and quality of life perceptions among residents. Journal of Travel Research, 50(3), 248-260.

https://doi.org/10.1177/0047287510362918

4. Andereck, K. L., y Vogt, C. A. (2000). The relationship between residents' attitudes toward tourism and tourism development options. Journal of Travel research, 39(1), 27-36. DOI: https://doi.org/10.1177/004728750003900104

5. Andereck, K. L., Valentine, K. M., Knopf, R. C., y Vogt, C. A. (2005). Residents' perceptions of community tourism impacts. Annals of tourism research, 32(4), 1056-1076. DOI: https://doi.org/10.1016/j.annals.2005.03.001

6. Añó, V. (2011). La Organización de Eventos y Competiciones Deportivas. Valencia: Universidad de Valencia.

7. Ap, J., y Crompton, J. L. (1998). Developing and testing a tourism impact scale. Journal of Travel Research, 37(2), $\quad 120-130 . \quad$ DOI: https://doi.org/10.1177/004728759803700203

8. Baron, R. M., y Kenny, D. A. (1986). The moderatormediator variable distinction in social psychological research: Conceptual, strategic, and statistical considerations. Journal of Personality and Social Psychology, 51(6), 1173-1182. DOI: http://dx.doi.org/10.1037/0022-3514.51.6.1173

9. Bartolomé, A., Ramos, V., y Rey-Maquieira, J. (2009). Residents' Attitudes Towards Diversification Sports Tourism in the Balearics. Tourism Recreation Research, 34(1), 55-65. DOI: https://doi.org/10.1080/02508281.2009.11081575

10. Brida, J. G., Del Chiappa, G., Meleddu, M., y Pulina, M. (2014). A comparison of residents' perceptions in two cruise ports in the Mediterranean Sea. 


\section{Percepciones de los residentes sobre el turismo deportivo}

International Journal of Tourism Research, 16(2), 180190. DOI: https://doi.org/10.1002/jtr.1915

11. Colmenares, L. (2008). La actitud de los residentes: factor clave en el desarrollo de un nuevo destino. Revista Scientia Unellezea, 3(1), 43-79.

12. Cooper, C. (1995). Strategic planning for sustainable tourism: the case of the offshore islands of the UK. Journal of Sustainable Tourism, 3(4), 191-209. DOI: https://doi.org/10.1080/09669589509510726

13. Chandralal, K. (2010). Impacts of tourism and community attitudes towards tourism: A case study in Sri Lanka. South Asian Journal of Tourism and Heritage, 3(2), 41-49.

14. Chen, S. C., y Raab, C. (2009). Measuring resident reactions to community tourism development: A pilot study of a new conceptual framework. Proceedings of the Hospitality and Tourism Management International CHRIE Conference, University of Massachusetts, Amherst. Recuperado en: https://scholarworks.umass.edu/cgi/viewcontent.cgi?re ferer=https://www.google.com/\&httpsredir=1\&article $=1202 \&$ context $=$ refereed

15. Fernández-Muñoz, J. J., y García-González, J. M. (2017). El análisis de mediación a través de la macro/interfaz Process para SPSS. REIRE Revista d'Innovació i Recerca en Educació, 10 (2), 79-88. Doi: http://doi.org/10.1344/reire2017.10.218109

16. Fredline, E. (2005). Host and guest relations and sport tourism. Sport in Society, 8(2), 263-279. DOI: https://doi.org/10.1080/17430430500087328

17. Fredline, E., Raybould, M., Jago, L., y Deery, M. (2005). Triple bottom line event evaluation: A proposed framework for jolistic event evaluation. En: J. Allen (Ed.), presented at the Event management research conference, Sydney. University of Technology, Sydney.

18. Gibson, H. (2017). Sport tourism and theory and other developments: some reflections. Journal of Sport \& Tourism, 2l(2) 153-158.

DOI: https://doi.org/10.1080/14775085.2017.1319514

19. González-García, R. J., Añó-Sanz, V., Parra-Camacho, D., y Calabuig-Moreno, F. (2018). Perception of residents about the impact of sports tourism on the community: Analysis and scale-validation. Journal of Physical Education and Sport, 18(1), 149-156. DOI: 10.7752/jpes.2018.01019

20. González-García, R. J., Parra, D., Añó, V. y Calabuig, F. (2016). Percepción de los residentes sobre el impacto del Mundobasket 2014 en Gran Canaria y apoyo a la celebración de eventos deportivos. Revista Iberoamericana de Psicología del Ejercicio y el Deporte, 11(2), 279-288.

21. Gursoy, D., Chi, C. G., y Dyer, P. (2010). Locals' attitudes toward mass and alternative tourism: The case of Sunshine Coast, Australia. Journal of Travel
Research, 49(3), 381-394. DOI: https://doi.org/10.1177/0047287509346853

22. Gursoy, D., Yolal, M., Ribeiro, M. A., y Panosso Netto, A. (2017). Impact of trust on local residents' megaevent perceptions and their support. Journal of Travel Research, 56(3), 393-406. DOI: https://doi.org/10.1177/0047287516643415

23. Hair, J.F., Anderson, R.E., Tatham, R.L. y Black, W.C. (2006). Multivariate Data Analysis, 5th ed., PrenticeHall, Upper Saddle River, NJ.

24. Hao, H., Long, P., y Kleckley, J. (2011). Factors predicting homeowners' attitudes toward tourism: A case of a coastal resort community. Journal of Travel research, 50(6), 627-640. DOI: https://doi.org/10.1177/0047287510385463

25. Hayes, A. F. (2013). Introduction to Mediation, Moderation, and Conditional Process Analysis: A Regression-Based Approach. New York, NY: The Guilford Press.

26. Hritz, N., y Ross, C. (2010). The perceived impacts of sport tourism: An urban host community perspective. Journal of Sport Management, 24(2), 119-138. DOI: https://doi.org/10.1123/jsm.24.2.119

27. Huh, C., y Vogt, C. A. (2008). Changes in residents' attitudes toward tourism over time: A cohort analytical approach. Journal of Travel Research, 46(4), 446-455. DOI: https://doi.org/10.1177/0047287507308327

28. Kaplanidou, K. (2012). The importance of legacy outcomes for Olympic Games four summer host cities residents' quality of life: 1996-2008. European Sport Management Quarterly, 12(4), 397-433. DOI: https://doi.org/10.1080/16184742.2012.693118

29. Kaplanidou, K., Karadakis, K., Gibson, H., Thapa, B., Walker, M., Geldenhuys, S., y Coetzee, W. (2013). Quality of life, event impacts, and mega-event support among South African residents before and after the 2010 FIFA world cup. Journal of Travel Research, 52(5), 631-645. DOI: https://doi.org/10.1177/0047287513478501

30. Kerstetter, D. L., y Bricker, K. S. (2012). Relationship between carrying capacity of small island tourism destinations and quality-of-life. En Handbook of tourism and quality-of-life research (pp. 445-462). Springer, Dordrecht.

31. Kim, K. (2002). The effects of tourism impacts upon quality of life of residents in the community (Tesis doctoral). Virginia Tech. Virginia.

32. Kim, K., Uysal, M., y Sirgy, M. J. (2013). How does tourism in a community impact the quality of life of community residents?. Tourism Management, 36, 527540.

DOI: https://doi.org/10.1016/j.tourman.2012.09.005

33. Ko, D. W., y Stewart, W. P. (2002). A structural equation model of residents' attitudes for tourism 


\section{González-García, R.J., Escamilla-Fajardo, P., López-Carril, S., Nuñez-Pomar, J.}

development. Tourism Management, 23(5), 521-530. DOI: https://doi.org/10.1016/S0261-5177(02)00006-7

34. Kreag, D. (2001). The Impacts of Tourism. Minnesota: Sea Grant Program.

35. Krippendorf, J. (1987). Ecological approach to tourism marketing. Tourism Management, 8(2), 174-176. DOI: https://doi.org/10.1016/0261-5177(87)90029-X

36. Látková, P., y Vogt, C. A. (2012). Residents' attitudes toward existing and future tourism development in rural communities. Journal of Travel Research, 51(1), 50-67.DOI: 10.1177/0047287510394193

37. Lee, T. H. (2013). Influence analysis of community resident support for sustainable tourism development. Tourism Management, 34, 37-46. DOI: https://doi.org/10.1016/j.tourman.2012.03.007

38. Liburd, J. J., Benckendorff, P., y Carlsen, J. (2012). Tourism and Quality-of-Life: How Does Tourism Measure Up?. En Handbook of Tourism and Qualityof-Life Research (pp. 105-132). Springer, Dordrecht.

39. Ma, S. C., y Kaplanidou, K. (2017). Legacy perceptions among host Tour de Taiwan residents: the mediating effect of quality of life. Leisure Studies, 36(3), 423437.

DOI: https://doi.org/10.1080/02614367.2015.1128475

40. Moscardo, G. (2012). Building social capital to enhance the Quality-of-life of destination residents. In Handbook of tourism and quality-of-life research (pp. 403-421). Springer, Dordrecht.

41. Müller, M. (2012). Popular perception of urban transformation through megaevents: understanding support for the 2014 Winter Olympics in Sochi. Environment and Planning C: Government and Policy, 30(4), 693-711. DOI: https://doi.org/10.1068/c11185r

42. Nunkoo, R., y Gursoy, D. (2012). Residents' support for tourism: An identity perspective. Annals of Tourism Research, 39(1), 243-268. DOI: https://doi.org/10.1016/j.annals.2011.05.006

43. Nunkoo, R., y Smith S. L. (2013). Political Economy of Tourism: Trust in Government Actors, Political Support, and Their Determinants. Tourism Management, $\quad 36, \quad 120-132 . \quad$ DOI: https://doi.org/10.1016/j.tourman.2012.11.018

44. Nunkoo, R., y So, K. K. F. (2016). Residents' support for tourism: Testing alternative structural models. Journal of Travel Research, 55(7), 847-861. DOI: https://doi.org/10.1177/0047287515592972

45. Parra, D., Añó, V., Calabuig, F., y Ayora, D. (2016). Percepción de los residentes sobre el legado de la America's Cup. Cuadernos de Psicología del Deporte, 16(1), 309-322.

46. Peters, M., y Schuckert, M. (2014). Tourism entrepreneurs' perception of quality of life: an explorative study. Tourism Analysis, 19(6), 731-740.
DOI:

https://doi.org/10.3727/108354214X14146846679484.

47. Plog, S. (2001). Why destination areas rise and fall in popularity: An update of a Cornell Quarterly classic. Cornell Hotel and Restaurant Administration Quarterly, 42(3), 13-24. DOI: https://doi.org/10.1016/S0010-8804(01)81020X

48. Rasoolimanesh, S. M., Jaafar, M., Kock, N., y Ramayah, T. (2015). A revised framework of social exchange theory to investigate the factors influencing residents' perceptions. Tourism Management Perspectives, 16, 335-345. DOI: https://doi.org/10.1016/j.tmp.2015.10.001

49. Ritchie, B. W., Burns, P. M., y Palmer, C. A. (Eds.). (2005). Tourism research methods: integrating theory with practice. Wallingford: Cabi Publishing.

50. Sharpley, R. (2014). Host perceptions of tourism: A review of the research. Tourism Management, 42, 3749.

DOI: https://doi.org/10.1016/j.tourman.2013.10.007

51. Sinclair-Maragh, G. (2017). Demographic analysis of residents' support for tourism development in Jamaica. Journal of Destination Marketing \& Management, 6(1), 5-12. DOI: https://doi.org/10.1016/j.jdmm.2016.03.005

52. Sinclair-Maragh, G., y Gursoy, D. (2016). A conceptual model of residents' support for tourism development in developing countries. Tourism Planning \& Development, 13(1), 1-22. DOI: https://doi.org/10.1080/21568316.2015.1047531

53. Sobel, M. E. (1982). Asymptotic confidence intervals for indirect effects in structural equation models. Sociological methodology, 13, 290-312.

54. Stylidis, D., Biran, A., Sit, J., y Szivas, E. M. (2014). Residents' support for tourism development: The role of residents' place image and perceived tourism impacts. Tourism Management, 45, 260-274. DOI: https://doi.org/10.1016/j.tourman.2014.05.006

55. Suess, C. (2014). Factors Influencing the Perceived Impacts of Medical Tourism Development on Quality of Life. (Tesis Doctoral). Universidad de Nevada. Las Vegas.

56. Twynam, G. y Johnston, M. (2004). Changes in Host Community Reactions to a Special Sporting Event. Current Issues in Tourism, 7(3), 242-261. DOI: https://doi.org/10.1080/13683500408667981

57. Uysal, M., Perdue, R., y Sirgy, M. J. (Eds.). (2012). Handbook of tourism and quality-of-life research: Enhancing the lives of tourists and residents of host communities. Springer Science \& Business Media.

58. Uysal, M., Sirgy, M. J., Woo, E., y Kim, H. L. (2016). Quality of life (QOL) and well-being research in 


\section{Percepciones de los residentes sobre el turismo deportivo}

tourism. Tourism Management, 53, 244-261. DOI: https://doi.org/10.1016/j.tourman.2015.07.013

59. Wang, Y., y Pfister, R. E. (2008). Residents' attitudes toward tourism and perceived personal benefits in a rural community. Journal of Travel Research, 47(1), 84-93. DOI: $10.1177 / 0047287507312402$

60. Weed, M. (2008). Sport y tourism: A reader. London: Routledge.

61. Williams, J., y Lawson, R. (2001). Community issues and resident opinions of tourism. Annals of Tourism Research, 28(2), 269-290. DOI: https://doi.org/10.1016/S0160-7383(00)00030-X

62. Woo, E., Kim, H., y Uysal, M. (2015). Life satisfaction and support for tourism development. Annals of Tourism Research, 50, 84-97. DOI: https://doi.org/10.1016/j.annals.2014.11.001

63. Woo, E., Uysal, M., y Sirgy, M. J. (2018). Tourism impact and stakeholders' quality of life. Journal of Hospitality \& Tourism Research, 42(2), 260-286. DOI: https://doi.org/10.1177/1096348016654971

64. Wood, J. F. (2011). An Introduction to Intercultural Communication: Identities in a Global Community. Journal of Intercultural Communication Research, 40(2), 173-175. DOI: https://doi.org/10.1080/17475759.2011.581035

65. Woosnam, K.M., y Aleshinloye, K.D. (2018). Residents' emotional solidarity with tourists: Explaining perceived impacts of a cultural heritage festival. Journal of Hospitality \& Tourism Research, 42(4), 587-605 DOI: 10.1177/1096348015584440

66. Xu, S., Barbieri, C., Anderson, D., Leung, Y. F., y Rozier-Rich, S. (2016). Residents' perceptions of wine tourism development. Tourism Management, 55, 276286.DOI:

https://doi.org/10.1016/j.tourman.2016.02.016

67. Yu, C. P., Chancellor, H. C., y Cole, S. T. (2011). Measuring residents' attitudes toward sustainable tourism: A reexamination of the sustainable tourism attitude scale. Journal of Travel Research, 50(1), 5763. DOI: https://doi.org/10.1177/0047287509353189

68. Zhao, X., Lynch Jr, J. G., y Chen, Q. (2010). Reconsidering Baron and Kenny: Myths and truths about mediation analysis. Journal of Consumer Research, 37(2), 197-206. DOI: https://doi.org/10.1086/651257 